
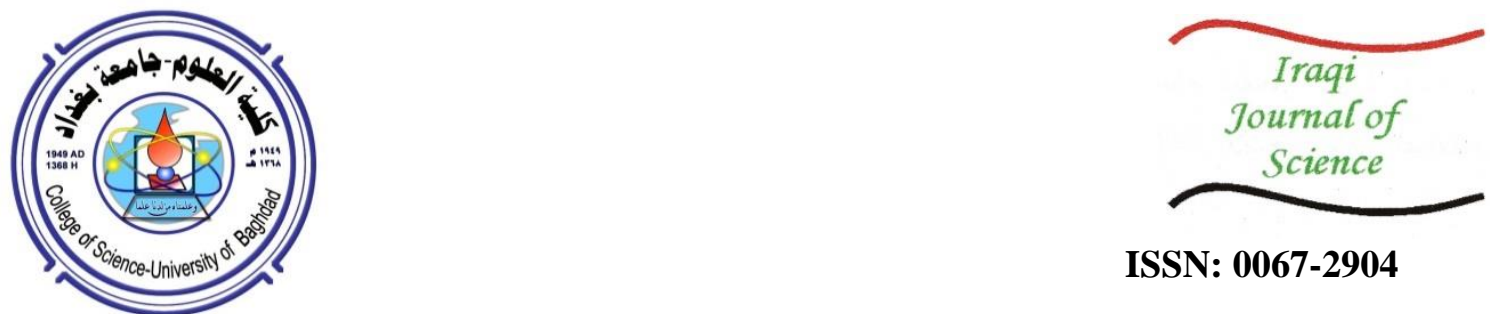

ISSN: 0067-2904

\title{
Estimating the Reliability Function for Transmuted Pareto Distribution Using Simulation
}

\author{
Masa Mohammed Hasan*, Makki A. Mohammed Salih \\ Department of Mathematics, College of Education, Almustansiriyah University, Iraq
}

Received: $14 / 2 / 2021$

Accepted: $18 / 4 / 2021$

\begin{abstract}
In this work, the methods (Moments, Modified Moments, L-Moments, Percentile, Rank Set sampling and Maximum Likelihood) were used to estimate the reliability function and the two parameters $(\alpha, \lambda)$ of the Transmuted Pareto (TP) distribution. We use simulation to generate the required data from three cases this indicates sample size $n=10,30,70,100$, and it replicates $N=1000$ for the real value for parameters, for reliability times values we take $t_{(i)}=0.1,0.2, \ldots \leq x_{(n)}$. Results were compared by using mean square error (MSE), the result appears as follows :

The best methods are Modified Moments, Maximum likelihood and L-Moments in first case, second case and third case respectively.
\end{abstract}

Keywords: Transmuted Pareto Distribution, Estimation methods, Simulation, Mean square error.

\section{تقدير دالة المعولية لتوزيع باريتو المحول باستخدام المحاكاة

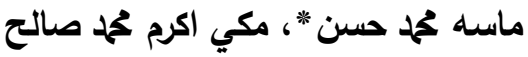 \\ قسم الرياضيات، كلية التربية، الجامعة المستصرية، بغداد، العراق الكر هلاق}

في هذا البحث ، تم استخدام طرائق (العزوم ، العزوم المطورة ، ل-العزوم ، النسبة المئوية ، رتبة مجموعة

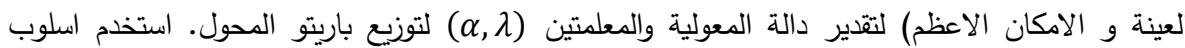

المحاكاة لإنشاء البيانات المطلوبة في ثلاث حالات من خلال حجم العينة

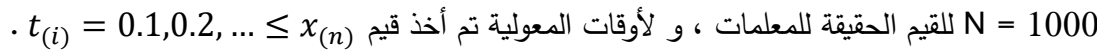

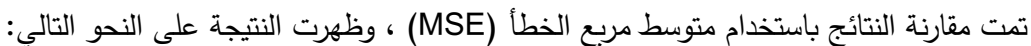

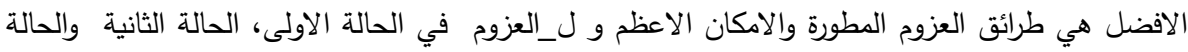

الثالثة على الترتيب.

\section{Introduction}

Vilfredo Pareto [1] suggested the Pareto distribution, the (pdf) and (cdf) of the Pareto distribution, respectively.

$$
\begin{aligned}
& g\left(x ; \alpha, x_{0}\right)=\frac{\alpha x_{0}^{\alpha}}{x^{\alpha+1}}, \\
& G\left(x ; \alpha, x_{0}\right)=1-\left(\frac{x_{0}}{x}\right)^{\alpha},
\end{aligned}
$$

\footnotetext{
*Email: masamohammed199@uomustansiriyah.edu.iq
} 
Where $X, \alpha>0$, and $0<\mathrm{x}_{0}<\mathrm{x}_{(1)}$.

Shaw and Buckley [2] introduced the Quadratic Rank Transmutation Map (QRTM) that the relationship satisfies the cumulative distribution function (cdf) approach.

$F(x)=(1+\lambda) G(x)-\lambda G^{2}(x),|\lambda| \leq 1$,

$f(x)=g(x)[1+\lambda-2 \lambda G(x)]$

Where $G(x)$ is the (cdf), and $g(x)$ is the (pdf) of the base distribution.

Several authors used the quadratic rank transmutation map to find generalizations to some distributions. Some of them are Aryal and Tsokos [3] proposed the transmuted Weibull distribution. Aryal [4] proposed the transmuted log-logistic distribution. Haq [5] introduced transmuted exponentiated inverse Rayleigh distribution. Vardhan and Balaswamy [6] proposed a transmuted new modified Weibull distribution. Elgarhy et al. [7] proposed transmuted generalized quasi Lindley distribution.

Faton and Llukan [8] proposed a transmuted Pareto distribution based on a Pareto distribution and a quadratic transmutation map.

In this paper, we will estimate the parameters and reliability function of the transmuted Pareto (TP) distribution through six methods which are not previously used in estimating by using simulation.

\section{Transmuted Pareto distribution (TP):}

Let $\mathrm{X}$ be a random variable of the (TP) distribution, then both of the (pdf) and (cdf) for the (TP) distribution are expressed respectively as [8].

$$
\begin{aligned}
& f_{T P}\left(x ; x_{0}, \alpha, \lambda\right)=\frac{\alpha x_{0}^{\alpha}}{x^{\alpha+1}}\left[1-\lambda+2 \lambda\left(\frac{x_{0}}{x}\right)^{\alpha}\right] \\
& F_{T P}\left(x ; x_{0}, \alpha, \lambda\right)=\left[1-\left(\frac{x_{0}}{x}\right)^{\alpha}\right]\left[1+\lambda\left(\frac{x_{0}}{x}\right)^{\alpha}\right]
\end{aligned}
$$

Where:

$x:$ is a value of random variable and $x>x_{0}$.

. Shape parameter and $\alpha>0: \alpha$

Transmutation parameter $|\lambda| \leq 1 .: \lambda$

$x_{0}$ : The minimum possible value of $x$.

The reliability function is given by

$R(t)=\left(\frac{x_{0}}{t}\right)^{\alpha}\left(\lambda\left(\frac{x_{0}}{t}\right)^{\alpha}-\lambda+1\right)$

Inverse distribution for (TP) distribution is

$x\left(p_{i}\right)=x_{0}\left[\frac{\lambda-1+\sqrt{(1-\lambda)^{2}-4 \lambda\left(p_{i}-1\right)}}{2 \lambda}\right]^{\frac{-1}{\alpha}}$

The $r^{\text {th }}$ order moment

$\mu_{r}=\mathrm{E}\left(x^{r}\right)=\frac{\alpha x_{0}^{r}(\alpha-r(1+\lambda))}{(\alpha-r)(2 \alpha-r)}, \quad \alpha>r$

When, $r=1,2$, the variance is

$$
\begin{aligned}
& \sigma^{2}=\operatorname{var}(x)=E\left(x^{2}\right)-(E(x))^{2} \\
&=\frac{\alpha x_{0}^{2}(\alpha-1-\lambda)}{(\alpha-1)(\alpha-2)}-\left(\frac{\alpha x_{0}(2 \alpha-1-\lambda)}{(\alpha-1)(2 \alpha-1)}\right)^{2} \\
& \sigma^{2}=\frac{\alpha x_{0}^{2}}{\alpha-1}\left[\frac{\alpha-1-\lambda}{\alpha-2}-\frac{\alpha(2 \alpha-1-\lambda)^{2}}{(\alpha-1)(2 \alpha-1)^{2}}\right], \alpha>2
\end{aligned}
$$

\section{Estimation Methods:}

In this section, we will derive some estimation methods for Transmuted Pareto distribution (TP). 


\subsection{Estimate Initial Value for Estimators:}

Makki and Jaafer [9] proposed a method for finding initial values for estimating parameters of any distribution, as follows:

The median of (TP) distribution that is given in [8] as follows :

$$
\begin{aligned}
& x_{\text {med }}=x_{0}\left[\frac{\lambda-1+\sqrt{1+\lambda^{2}}}{2 \lambda}\right]^{\frac{-1}{\alpha}} \\
& \frac{x_{\text {med }}}{x_{0}}=\left[\frac{\lambda-1+\sqrt{1+\lambda^{2}}}{2 \lambda}\right]^{\frac{-1}{\alpha}}
\end{aligned}
$$

Taking the natural logarithm for the equation (6), getting

$$
\begin{aligned}
& \ln \left(\frac{x_{\text {med }}}{x_{0}}\right)=\frac{-1}{\alpha} \ln \left[\frac{\lambda-1+\sqrt{1+\lambda^{2}}}{2 \lambda}\right] \\
& \hat{\alpha}_{0}=\frac{-\ln \left[\frac{\lambda-1+\sqrt{1+\lambda^{2}}}{2 \lambda}\right]}{\ln \left(\frac{x_{m e d}}{x_{0}}\right)}
\end{aligned}
$$

From equation (6)

$$
\begin{aligned}
& \left(\frac{x_{m e d}}{x_{0}}\right)^{-\alpha}=\frac{\lambda-1+\sqrt{1+\lambda^{2}}}{2 \lambda} \\
& \hat{\lambda}_{0}=\frac{\lambda-1+\sqrt{1+\lambda^{2}}}{2\left(\frac{x_{m e d}}{x_{0}}\right)^{-\alpha}}
\end{aligned}
$$

We can use $\hat{\alpha}_{0}$ and $\hat{\lambda}_{0}$ in equations (7) and (8) are the initial value to another parameter estimator formula. $x_{\text {med }}$ can be obtained from the generating sample.

\subsection{Moments Method (MOM):}

The idea of this method is to equate the moments of the distribution with the moments of the sample.

$\mu_{r}=\grave{\mu}_{r} \quad, r=1,2$

The first estimate can be get it when $(r=1)$ in equation (9), then

$\mu_{1}=\grave{\mu}_{1}$

Since,

$E(x)=\frac{\alpha x_{0}(2 \alpha-1-\lambda)}{(\alpha-1)(2 \alpha-1)}=\frac{\sum_{i=1}^{n} x_{i}}{n}=\bar{x}$

$2 \alpha-1-\lambda=\frac{\bar{x}(\alpha-1)(2 \alpha-1)}{\alpha x_{0}}$

$\hat{\lambda}_{M O M}=2 \alpha_{0}-1-\frac{\bar{x}\left(\alpha_{0}-1\right)\left(2 \alpha_{0}-1\right)}{\alpha_{0} x_{0}}$

The second estimate can be get it when $(r=2)$ in equation (9) then

$\mu_{2}=\grave{\mu}_{2}$

Since

$E\left(x^{2}\right)=\frac{\alpha x_{0}^{2}(\alpha-1-\lambda)}{(\alpha-1)(\alpha-2)}=\frac{\sum_{i=1}^{n} x_{i}^{2}}{n}$ 


$$
\begin{aligned}
& (\alpha-2)=\frac{\alpha x_{0}^{2}(\alpha-1-\lambda)}{\frac{\sum_{i=1}^{n} x_{i}^{2}}{n}(\alpha-1)} \\
& \hat{\alpha}_{M O M}=\frac{\alpha_{0} x_{0}^{2}\left(\alpha_{0}-1-\lambda_{0}\right)}{\frac{\sum_{i=1}^{n} x_{i}^{2}}{n}\left(\alpha_{0}-1\right)}+2
\end{aligned}
$$

As for the estimator of the approximate reliability function for this distribution, we obtain it by substituting equations (10) and (11) into equation (3) as follows:

$\hat{R}(t)_{M O M}=\left(\frac{x_{0}}{t}\right)^{\hat{\alpha}_{M O M}}\left(\hat{\lambda}_{M O M}\left(\frac{x_{0}}{t}\right)^{\hat{\alpha}_{M O M}}-\hat{\lambda}_{M O M}+1\right)$

\subsection{Modified Moments Method (MM):}

Cohen and Whitten [10] suggested a new modified, they used the following equation.

$E\left(\widehat{F}\left(x_{(i)}\right)\right)=F\left(x_{(i)}\right)$

Where $i$ represents the order of observations that arrange in ascending orders. $\hat{F}\left(x_{(i)}\right)$ is estimated unbiased for function distribution $F\left(x_{(i)}\right)$, and $F\left(x_{(i)}\right)$ is replaced by plotting position formula [11].

$$
\begin{aligned}
& P_{i}=\frac{i}{n+1}, \mathrm{i}=1,2, \ldots, \mathrm{n} \\
& F\left(x_{i}\right)=\frac{i}{n+1}, \mathrm{i}=1,2, \ldots, \mathrm{n} \\
& F\left(x_{(1)}\right)=\frac{1}{n+1}
\end{aligned}
$$

From equations (2), and (15), we get:

$$
\begin{aligned}
& {\left[1-\left(\frac{x_{0}}{x_{(1)}}\right)^{\alpha}\right]\left[1+\lambda\left(\frac{x_{0}}{x_{(1)}}\right)^{\alpha}\right]=\frac{1}{n+1}} \\
& 1+\lambda\left(\frac{x_{0}}{x_{(1)}}\right)^{\alpha}-\left(\frac{x_{0}}{x_{(1)}}\right)^{\alpha}-\lambda\left(\frac{x_{0}}{x_{(1)}}\right)^{2 \alpha}=\frac{1}{n+1} \\
& \lambda\left(\frac{x_{0}}{x_{(1)}}\right)^{\alpha}-\left(\frac{x_{0}}{x_{(1)}}\right)^{\alpha}-\lambda\left(\frac{x_{0}}{x_{(1)}}\right)^{2 \alpha}=\frac{-n}{n+1} \\
& \lambda\left(\frac{x_{0}}{x_{(1)}}\right)^{\alpha}=\left(\frac{x_{0}}{x_{(1)}}\right)^{\alpha}+\lambda\left(\frac{x_{0}}{x_{(1)}}\right)^{2 \alpha}-\frac{n}{n+1} \\
& \hat{\lambda}_{M M}=\frac{\left(\frac{x_{0}}{x_{(1)}}\right)^{\alpha_{0}}+\lambda_{0}\left(\frac{x_{0}}{x_{(1)}}\right)^{2 \alpha_{0}}-\frac{n}{n+1}}{\left(\frac{x_{0}}{x_{(1)}}\right)^{\alpha_{0}}}
\end{aligned}
$$

Since,

$$
\operatorname{var}(x)=E\left(x^{2}\right)-(E(x))^{2}=\frac{\sum_{i=1}^{n}\left(x_{i}-\bar{x}\right)^{2}}{n-1}
$$

Substitute equation (5) in equation (17), we get

$$
\begin{aligned}
& \frac{\alpha x_{0}^{2}}{(\alpha-1)}\left[\frac{\alpha-1-\lambda}{\alpha-2}-\frac{\alpha(2 \alpha-1-\lambda)^{2}}{(\alpha-1)(2 \alpha-1)^{2}}\right]=\frac{\sum_{i=1}^{n}\left(x_{i}-\bar{x}\right)^{2}}{n-1} \\
& \frac{\sum_{i=1}^{n}\left(x_{i}-\bar{x}\right)^{2}}{(n-1)} \frac{(\alpha-1)}{\alpha x_{0}^{2}}=\frac{\alpha-1-\lambda}{\alpha-2}-\frac{\alpha(2 \alpha-1-\lambda)^{2}}{(\alpha-1)(2 \alpha-1)^{2}}
\end{aligned}
$$




$$
\begin{aligned}
& (\alpha-2)\left(\frac{\sum_{i=1}^{n}\left(x_{i}-\bar{x}\right)^{2}}{(n-1)} \frac{(\alpha-1)}{\alpha x_{0}^{2}}+\frac{\alpha(2 \alpha-1-\lambda)^{2}}{(\alpha-1)(2 \alpha-1)^{2}}\right)=\alpha-1-\lambda \\
& \hat{\alpha}_{M M}=\frac{\alpha_{0}-1-\lambda_{0}}{\frac{\sum_{i=1}^{n}\left(x_{i}-\bar{x}\right)^{2}}{(n-1)} \frac{\left(\alpha_{0}-1\right)}{\alpha_{0} x_{0}^{2}}+\frac{\alpha_{0}\left(2 \alpha_{0}-1-\lambda_{0}\right)^{2}}{\left(\alpha_{0}-1\right)\left(2 \alpha_{0}-1\right)^{2}}}+2
\end{aligned}
$$

As for the estimator of the approximate reliability function for this distribution, we obtain it by substituting equations (16) and (18) into equation (3) as follows:

$\hat{R}(t)_{M M}=\left(\frac{x_{0}}{t}\right)^{\hat{\alpha}_{M M}}\left(\hat{\lambda}_{M M}\left(\frac{x_{0}}{t}\right)^{\hat{\alpha}_{M M}}-\hat{\lambda}_{M M}+1\right)$

\subsection{L-Moment Method (LM):}

Hosking [12] proposed the L-Moments method. The L-Moments methods are anticipations of certain linear combinations of order statistics[13]. The distribution parameters can be estimated by the following

$$
B_{r}=\int_{x_{0}}^{\infty} x\left(F^{r}\right) d F
$$

Substitution equations (1) and (2) into equation (20), then we get

$$
B_{r}=\int_{x_{0}}^{\infty} x\left[\left[1-\left(\frac{x_{0}}{x}\right)^{\alpha}\right]\left[1+\lambda\left(\frac{x_{0}}{x}\right)^{\alpha}\right]\right]^{r} \frac{\alpha x_{0}^{\alpha}}{x^{\alpha+1}}\left[1-\lambda+2 \lambda\left(\frac{x_{0}}{x}\right)^{\alpha}\right] d x
$$

If we substitute $r=0$ in equation (21), then we get

$$
\begin{aligned}
B_{0} & =\alpha x_{0}^{\alpha} \int_{x_{0}}^{\infty} \frac{1}{x^{\alpha}}\left[1-\lambda+2 \lambda x_{0}^{\alpha} \frac{1}{x^{\alpha}}\right] d x \\
& =\alpha x_{0}^{\alpha}\left[\frac{-1}{(\alpha-1) x^{\alpha-1}}+\frac{\lambda}{(\alpha-1) x^{\alpha-1}}-\left.\frac{2 \lambda x_{0}^{\alpha}}{(2 \alpha-1) x^{2 \alpha-1}}\right|_{x_{0}} ^{\infty}\right] \\
& =\frac{\alpha x_{0}^{\alpha}}{(\alpha-1) x_{0}^{\alpha-1}}-\frac{\alpha \lambda x_{0}^{\alpha}}{(\alpha-1) x_{0}^{\alpha-1}}+\frac{2 \alpha \lambda x_{0}^{2 \alpha}}{(2 \alpha-1) x_{0}^{2 \alpha-1}} \\
& =\frac{\alpha x_{0}}{\alpha-1}-\frac{\alpha \lambda x_{0}}{\alpha-1}+\frac{2 \alpha \lambda x_{0}}{2 \alpha-1} \\
B_{0} & =\alpha x_{0}\left[\frac{1-\lambda}{\alpha-1}+\frac{2 \lambda}{2 \alpha-1}\right]
\end{aligned}
$$

While if we substitute $r=1$ in equation (21), we get

$$
\begin{aligned}
B_{1} & =\int_{x_{0}}^{\infty} x\left[\left[1-\left(\frac{x_{0}}{x}\right)^{\alpha}\right]\left[1+\lambda\left(\frac{x_{0}}{x}\right)^{\alpha}\right]\right] \frac{\alpha x_{0}^{\alpha}}{x^{\alpha+1}}\left[1-\lambda+2 \lambda\left(\frac{x_{0}}{x}\right)^{\alpha}\right] d x \\
& =\alpha x_{0}^{\alpha} \int_{x_{0}}^{\infty} \frac{1}{x^{\alpha}}\left[1+\lambda x_{0}^{\alpha} \frac{1}{x^{\alpha}}-x_{0}^{\alpha} \frac{1}{x^{\alpha}}-\lambda x_{0}^{2 \alpha} \frac{1}{x^{2 \alpha}}\right]\left[1-\lambda+2 \lambda x_{0}^{\alpha} \frac{1}{x^{\alpha}}\right] d x \\
& =\alpha x_{0}^{\alpha} \int_{x_{0}}^{\infty}\left[1+\lambda x_{0}^{\alpha} \frac{1}{x^{\alpha}}-x_{0}^{\alpha} \frac{1}{x^{\alpha}}-\lambda x_{0}^{2 \alpha} \frac{1}{x^{2 \alpha}}\right]\left[\frac{1}{x^{\alpha}}-\frac{\lambda}{x^{\alpha}}+2 \lambda x_{0}^{\alpha} \frac{1}{x^{2 \alpha}}\right] d x \\
& =\alpha x_{0}^{\alpha} \int_{x_{0}}^{\infty}\left[\frac{1}{x^{\alpha}}-\frac{\lambda}{x^{\alpha}}+\frac{4 \lambda x_{0}^{\alpha}}{x^{2 \alpha}}-\frac{\lambda^{2} x_{0}^{\alpha}}{x^{2 \alpha}}+\frac{3 \lambda^{2} x_{0}^{2 \alpha}}{x^{3 \alpha}}-\frac{x_{0}^{\alpha}}{x^{2 \alpha}}-\frac{3 \lambda x_{0}^{2 \alpha}}{x^{3 \alpha}}-\frac{2 \lambda^{2} x_{0}^{3 \alpha}}{x^{4 \alpha}}\right] d x
\end{aligned}
$$




$$
\begin{aligned}
= & \alpha x_{0}^{\alpha}\left[\frac{-1}{(\alpha-1) x^{\alpha-1}}+\frac{\lambda}{(\alpha-1) x^{\alpha-1}}-\frac{4 \lambda x_{0}^{\alpha}}{(2 \alpha-1) x^{2 \alpha-1}}+\frac{\lambda^{2} x_{0}^{\alpha}}{(2 \alpha-1) x^{2 \alpha-1}}\right. \\
& \left.\quad-\frac{3 \lambda^{2} x_{0}^{2 \alpha}}{(3 \alpha-1) x^{3 \alpha-1}}+\frac{x_{0}^{\alpha}}{(2 \alpha-1) x^{2 \alpha-1}}+\frac{3 \lambda x_{0}^{2 \alpha}}{(3 \alpha-1) x^{3 \alpha-1}}+\left.\frac{2 \lambda^{2} x_{0}^{3 \alpha}}{(4 \alpha-1) x^{4 \alpha-1}}\right|_{x_{0}} ^{\infty}\right] \\
= & \frac{\alpha x_{0}^{\alpha}}{(\alpha-1) x_{0}^{\alpha-1}}-\frac{\alpha \lambda x_{0}^{\alpha}}{(\alpha-1) x_{0}^{\alpha-1}}+\frac{4 \alpha \lambda x_{0}^{2 \alpha}}{(2 \alpha-1) x_{0}^{2 \alpha-1}}-\frac{\alpha \lambda^{2} x_{0}^{2 \alpha}}{(2 \alpha-1) x_{0}^{2 \alpha-1}}+\frac{3 \alpha \lambda^{2} x_{0}^{3 \alpha}}{(3 \alpha-1) x_{0}^{3 \alpha-1}} \\
= & \frac{\alpha x_{0}}{\alpha-1}-\frac{\alpha \lambda x_{0}}{\alpha-1}+\frac{4 \alpha \lambda x_{0}}{2 \alpha-1}-\frac{\alpha \lambda^{2} x_{0}}{2 \alpha-1}+\frac{3 \alpha \lambda^{2} x_{0}}{3 \alpha-1}-\frac{\alpha x_{0}}{2 \alpha-1}-\frac{3 \alpha \lambda x_{0}}{3 \alpha-1}-\frac{2 \alpha \lambda^{2} x_{0}}{4 \alpha-1} \\
= & \frac{\alpha x_{0}(1-\lambda)}{\alpha-1}+\frac{\alpha x_{0}\left(4 \lambda-\lambda^{2}-1\right)}{2 \alpha-1}+\frac{\alpha x_{0}\left(3 \lambda^{2}-3 \lambda\right)}{3 \alpha-1}-\frac{2 \alpha x_{0} \lambda^{2}}{4 \alpha-1} \\
B_{1}= & \alpha x_{0}\left[\frac{1-\lambda}{\alpha-1}+\frac{4 \lambda-\lambda^{2}-1}{2 \alpha-1}+\frac{3 \lambda^{2}-3 \lambda}{3 \alpha-1}-\frac{2 \lambda^{2}}{4 \alpha-1}\right]
\end{aligned}
$$

Now subtract equation (23) from equation (22), we have

$$
\begin{aligned}
& B_{0}-B_{1}=\alpha x_{0}\left[\frac{1-2 \lambda+\lambda^{2}}{2 \alpha-1}+\frac{3 \lambda(\lambda-1)}{3 \alpha-1}-\frac{2 \lambda^{2}}{4 \alpha-1}\right] \\
& \hat{\alpha}_{L M}=\frac{B_{0}-B_{1}}{x_{0}\left[\frac{1-2 \lambda_{0}+\lambda_{0}^{2}}{2 \alpha_{0}-1}+\frac{3 \lambda_{0}\left(\lambda_{0}-1\right)}{3 \alpha_{0}-1}-\frac{2 \lambda_{0}^{2}}{4 \alpha_{0}-1}\right]}
\end{aligned}
$$

From equation (22), we get

$$
\begin{aligned}
& \frac{B_{0}}{\alpha x_{0}}-\frac{1-\lambda}{\alpha-1}=\frac{2 \lambda}{2 \alpha-1} \\
& \hat{\lambda}_{L M}=\frac{\left(2 \hat{\alpha}_{L M}-1\right)\left[\frac{B_{0}}{\hat{\alpha}_{L M} x_{0}}-\frac{1-\lambda_{0}}{\hat{\alpha}_{L M}-1}\right]}{2}
\end{aligned}
$$

As for the estimator of the approximate reliability function for this distribution, we obtain it by substituting equations (24) and (25) into the equation (3) as follows:

$\hat{R}(t)_{L m}=\left(\frac{x_{0}}{t}\right)^{\hat{\alpha}_{L M}}\left(\hat{\lambda}_{L M}\left(\frac{x_{0}}{t}\right)^{\hat{\alpha}_{L M}}-\hat{\lambda}_{L M}+1\right)$

\subsection{Percentile Estimation Method (PER):}

This method was originally explored by Kao [14]. This method depends on parameter estimation of any distribution on minimized of inverse distribution which represented from the equation (4) as follows:

$\frac{x\left(p_{i}\right)}{x_{0}}=\left[\frac{\lambda-1+\sqrt{(1-\lambda)^{2}-4 \lambda\left(p_{i}-1\right)}}{2 \lambda}\right]^{\frac{-1}{\alpha}}$

Taking the natural logarithm for equation (27), we get

$$
\ln \left(\frac{x\left(p_{i}\right)}{x_{0}}\right)=\frac{-1}{\alpha} \ln \left[\frac{\lambda-1+\sqrt{(1-\lambda)^{2}-4 \lambda\left(p_{i}-1\right)}}{2 \lambda}\right]
$$

We square the equation (28), and let it equal to zero, we get the following

$$
\sum_{i=1}^{n}\left(\ln \left(\frac{x\left(p_{i}\right)}{x_{0}}\right)+\frac{1}{\alpha} \ln \left[\frac{\lambda-1+\sqrt{(1-\lambda)^{2}-4 \lambda\left(p_{i}-1\right)}}{2 \lambda}\right]\right)^{2}=0
$$

The partial derivative the equation (29) with respect to $a$ is 


$$
\left.\left.\begin{array}{c}
\frac{\partial \delta}{\partial \alpha}=2 \sum_{i=1}^{n}\left(\begin{array}{c}
\left(\ln \left(\frac{x\left(p_{i}\right)}{x_{0}}\right)+\frac{1}{\alpha} \ln \left[\frac{\lambda-1+\sqrt{(1-\lambda)^{2}-4 \lambda\left(p_{i}-1\right)}}{2 \lambda}\right]\right. \\
\left(\frac{-1}{\alpha^{2}} \ln \left[\frac{\lambda-1+\sqrt{(1-\lambda)^{2}-4 \lambda\left(p_{i}-1\right)}}{2 \lambda}\right]\right)
\end{array}\right)=0 \\
\frac{1}{\alpha^{2}} \sum_{i=1}^{n} \ln \left[\frac{\lambda-1+\sqrt{(1-\lambda)^{2}-4 \lambda\left(p_{i}-1\right)}}{2 \lambda} \ln \left(\frac{x\left(p_{i}\right)}{x_{0}}\right)\right.
\end{array}\right)=\frac{-1}{\alpha^{3} \sum_{i=1}^{n}\left(\ln \left[\frac{\lambda-1+\sqrt{(1-\lambda)^{2}-4 \lambda\left(p_{i}-1\right)}}{2 \lambda}\right]\right)^{2}}\right)_{\sum_{i=1}^{n} \ln \left[\frac{\lambda_{0}-1+\sqrt{\left(1-\lambda_{0}\right)^{2}-4 \lambda_{0}\left(p_{i}-1\right)}}{2 \lambda_{0}}\right] \ln \left(\frac{x\left(p_{i}\right)}{x_{0}}\right)}^{-\sum_{i=1}^{n}\left(\operatorname { l n } \left[\frac{\lambda_{0}-1+\sqrt{\left(1-\lambda_{0}\right)^{2}-4 \lambda_{0}\left(p_{i}-1\right)}}{2 \lambda_{0}}\right.\right.}
$$

The partial derivative the equation (29) with respect to $\lambda$ is $\partial \delta$

$\overline{\partial \lambda}$

$$
\left.=2 \sum_{i=1}^{n}\left(\begin{array}{c}
\left(\ln \left(\frac{x\left(p_{i}\right)}{x_{0}}\right)+\frac{1}{\alpha} \ln \left[\frac{\lambda-1+\sqrt{(1-\lambda)^{2}-4 \lambda\left(p_{i}-1\right)}}{2 \lambda}\right]\right) \\
\frac{1}{\alpha}\left(\frac{2 \lambda}{\lambda-1+\sqrt{(1-\lambda)^{2}-4 \lambda\left(p_{i}-1\right)}}\right) \\
\left(\frac{2 \lambda\left(1+\frac{2(1-\lambda)(-1)-4\left(p_{i}-1\right)}{2 \sqrt{(1-\lambda)^{2}-4 \lambda\left(p_{i}-1\right)}}\right)-2\left(\lambda-1+\sqrt{(1-\lambda)^{2}-4 \lambda\left(p_{i}-1\right)}\right)}{4 \lambda^{2}}\right)
\end{array}\right)\right)
$$

$=0$

If we let $k_{i}=\sqrt{(1-\lambda)^{2}-4 \lambda\left(p_{i}-1\right)}$, then

$\sum_{i=1}^{n}\left(\left(\frac{\left.\ln \left(\frac{x\left(p_{i}\right)}{x_{0}}\right)+\frac{1}{\alpha} \ln \left[\frac{\lambda-1+k_{i}}{2 \lambda}\right]\right)}{\left.\frac{1}{\alpha}\left(\frac{2 \lambda}{\lambda-1+k_{i}}\right)\left(\frac{\lambda\left(1+\frac{\lambda-1-2\left(p_{i}-1\right)}{k_{i}}\right)-\left(\lambda-1+k_{i}\right)}{\lambda^{2}}\right)\right)}\right)=0\right.$

We multiply equation (31) by $\alpha$, then we have

$$
\begin{aligned}
& \sum_{i=1}^{n} \ln \left(\frac{x\left(p_{i}\right)}{x_{0}}\right)\left(\left(\frac{2 \lambda}{\lambda-1+k_{i}}\right)\left(\frac{\lambda\left(1+\frac{\lambda-1-2\left(p_{i}-1\right)}{k_{i}}\right)-\left(\lambda-1+k_{i}\right)}{\lambda^{2}}\right)\right) \\
& =\frac{-1}{\alpha} \sum_{i=1}^{n} \ln \left(\frac{\lambda-1+k_{i}}{2 \lambda}\right)\left(\left(\frac{2 \lambda}{\lambda-1+k_{i}}\right)\left(\frac{\lambda\left(1+\frac{\lambda-1-2\left(p_{i}-1\right)}{k_{i}}\right)-\left(\lambda-1+k_{i}\right)}{\lambda^{2}}\right)\right)
\end{aligned}
$$




$$
\begin{aligned}
\hat{\lambda}_{P E R}= & \left(-\alpha_{0} \lambda_{0} \sum_{i=1}^{n} \ln \left(\frac{x\left(p_{i}\right)}{x_{0}}\right)\left(\left(\frac{2 \lambda_{0}}{\lambda_{0}-1+k_{i}}\right)\left(\lambda_{0}\left(1+\frac{\lambda_{0}-1-2\left(p_{i}-1\right)}{k_{i}}\right)\right)-\left(\lambda_{0}-1+k_{i}\right)\right)\right) / \\
& \left(\sum_{i=1}^{n} \ln \left(\frac{\lambda_{0}-1+k_{i}}{2 \lambda_{0}}\right)\left(\left(\frac{2 \lambda_{0}}{\lambda_{0}-1+k_{i}}\right)\left(\lambda_{0}\left(1+\frac{\lambda_{0}-1-2\left(p_{i}-1\right)}{k_{i}}\right)\right)-\left(\lambda_{0}-1+k_{i}\right)\right)\right) \ldots(32)
\end{aligned}
$$

As for the estimator of the approximate reliability function for this distribution, we obtain it by substituting equations (30) and (32) into equation (3) as follows:

$\hat{R}(t)_{P E R}=\left(\frac{x_{0}}{t}\right)^{\widehat{\alpha}_{P E R}}\left(\hat{\lambda}_{P E R}\left(\frac{x_{0}}{t}\right)^{\hat{\alpha}_{P E R}}-\hat{\lambda}_{P E R}+1\right)$

\subsection{Rank Set Sampling Method (RSS):}

Let $\left(x_{1}, x_{2}, \ldots, x_{n}\right)$ be a random sample. Assumed that $\left(x_{(1)}, x_{(2)}, \ldots, x_{(n)}\right)$ is the order statistics that obtained by ordering the sample in increasing order [15].

The (pdf) of $x_{(i)}$ is:

$f\left(x_{i}\right)=\frac{n !}{(i-1) !(n-i) !}\left[F\left(x_{(i)}\right)\right]^{i-1}\left[1-F\left(x_{(i)}\right)\right]^{n-i} f\left(x_{(i)}\right)$

By substitution equations (1) and (2) into equation (34), and let $Q_{i}=\frac{n !}{(i-1) !(n-i) !}$, getting

$$
\begin{gathered}
f\left(x_{i}\right)=Q_{i}\left[\left(1-\left(\frac{x_{0}}{x_{(i)}}\right)^{\alpha}\right)\left(1+\lambda\left(\frac{x_{0}}{x_{(i)}}\right)^{\alpha}\right)\right]^{i-1}\left[\left(\frac{x_{0}}{x_{(i)}}\right)^{\alpha}\left(\lambda\left(\frac{x_{0}}{x_{(i)}}\right)^{\alpha}-\lambda+1\right)\right]^{n-i} \\
\frac{\alpha x_{0}^{\alpha}}{x_{(i)}{ }^{\alpha+1}}\left[1-\lambda+2 \lambda\left(\frac{x_{0}}{x_{(i)}}\right)^{\alpha}\right]
\end{gathered}
$$

The likelihood for equation (35) is

$$
\begin{aligned}
L f=Q_{i}^{n} \prod_{i=1}^{n} & {\left[\left(1-\left(\frac{x_{0}}{x_{(i)}}\right)^{\alpha}\right)\left(1+\lambda\left(\frac{x_{0}}{x_{(i)}}\right)^{\alpha}\right)\right]^{i-1} \prod_{i=1}^{n}\left[\left(\frac{x_{0}}{x_{(i)}}\right)^{\alpha}(1-\lambda\right.} \\
& \left.\left.+\lambda\left(\frac{x_{0}}{x_{(i)}}\right)^{\alpha}\right)\right]^{n-i} \alpha^{n} x_{0}^{\alpha n} \prod_{i=1}^{n}\left[\frac{1-\lambda+2 \lambda\left(\frac{x_{0}}{x_{(i)}}\right)^{\alpha}}{x_{(i)}{ }^{\alpha+1}}\right]
\end{aligned}
$$

Taking the natural logarithm for the equation (36), we get

$$
\begin{aligned}
\ln L f=n \ln Q_{i} & +\sum_{i=1}^{n}(i-1) \ln \left[\left[1-\left(\frac{x_{0}}{x_{(i)}}\right)^{\alpha}\right]\left[1+\lambda\left(\frac{x_{0}}{x_{(i)}}\right)^{\alpha}\right]\right] \\
& +\sum_{i=1}^{n}(n-i) \ln \left[\left(\frac{x_{0}}{x_{(i)}}\right)^{\alpha}\left(1-\lambda+\lambda\left(\frac{x_{0}}{x_{(i)}}\right)^{\alpha}\right)\right]+n \ln \alpha+n \alpha \ln x_{0} \\
& +\sum_{i=1}^{n} \ln \left(1-\lambda+2 \lambda\left(\frac{x_{0}}{x_{(i)}}\right)^{\alpha}\right)-(\alpha+1) \sum_{i=1}^{n} \ln x_{(i)}
\end{aligned}
$$




$$
\begin{aligned}
\ln L f=n \ln Q_{i} & +\sum_{i=1}^{n}(i-1) \ln \left(1-\left(\frac{x_{0}}{x_{(i)}}\right)^{\alpha}\right)+\sum_{i=1}^{n}(i-1) \ln \left(1+\lambda\left(\frac{x_{0}}{x_{(i)}}\right)^{\alpha}\right) \\
& +\alpha \sum_{i=1}^{n}(n-i) \ln \left(\frac{x_{0}}{x_{(i)}}\right)+\sum_{i=1}^{n}(n-i) \ln \left(1-\lambda+\lambda\left(\frac{x_{0}}{x_{(i)}}\right)^{\alpha}\right)+n \ln \alpha \\
& +n \alpha \ln x_{0}-\alpha \sum_{i=1}^{n} \ln x_{(i)}-\sum_{i=1}^{n} \ln x_{(i)} \\
& +\sum_{i=1}^{n} \ln \left(1-\lambda+2 \lambda\left(\frac{x_{0}}{x_{(i)}}\right)^{\alpha}\right) . .(37)
\end{aligned}
$$

We take partial derivative for the equation (37) with respect to $\alpha$, and put it equal to zero

$$
\begin{aligned}
& \frac{\partial \ln l f}{\partial \alpha}=-\sum_{i=1}^{n}(i-1) \frac{\left(\frac{x_{0}}{x_{(i)}}\right)^{\alpha} \ln \left(\frac{x_{0}}{x_{(i)}}\right)}{1-\left(\frac{x_{0}}{x_{(i)}}\right)^{\alpha}}+\sum_{i=1}^{n}(i-1) \frac{\lambda\left(\frac{x_{0}}{x_{(i)}}\right)^{\alpha} \ln \left(\frac{x_{0}}{x_{(i)}}\right)}{1+\lambda\left(\frac{x_{0}}{x_{(i)}}\right)^{\alpha}} \\
& +\sum_{i=1}^{n}(n-i) \ln \left(\frac{x_{0}}{x_{(i)}}\right)+\sum_{i=1}^{n}(n-i) \frac{\lambda\left(\frac{x_{0}}{x_{(i)}}\right)^{\alpha} \ln \left(\frac{x_{0}}{x_{(i)}}\right)}{1-\lambda+\lambda\left(\frac{x_{0}}{x_{(i)}}\right)^{\alpha}}+\frac{n}{\alpha}+n \ln x_{0} \\
& -\sum_{i=1}^{n} \ln x_{(i)}+\sum_{i=1}^{n} \frac{2 \lambda\left(\frac{x_{0}}{x_{(i)}}\right)^{\alpha} \ln \left(\frac{x_{0}}{x_{(i)}}\right)}{1-\lambda+2 \lambda\left(\frac{x_{0}}{x_{(i)}}\right)^{\alpha}}=0 \\
& -x_{0}^{\alpha} \sum_{i=1}^{n}(i-1) \frac{\frac{1}{x^{\alpha}} \ln \left(\frac{x_{0}}{x_{(i)}}\right)}{1-\left(\frac{x_{0}}{x_{(i)}}\right)^{\alpha}}+x_{0}^{\alpha} \sum_{i=1}^{n}(i-1) \frac{\frac{\lambda}{x^{\alpha}} \ln \left(\frac{x_{0}}{x_{(i)}}\right)}{1+\lambda\left(\frac{x_{0}}{x_{(i)}}\right)^{\alpha}}+\sum_{i=1}^{n}(n-i) \ln \left(\frac{x_{0}}{x_{(i)}}\right) \\
& +x_{0}^{\alpha} \sum_{i=1}^{n}(n-i) \frac{\frac{\lambda}{x^{\alpha}} \ln \left(\frac{x_{0}}{x_{(i)}}\right)}{1-\lambda+\lambda\left(\frac{x_{0}}{x_{(i)}}\right)^{\alpha}}+\frac{n}{\alpha}+n \ln x_{0}-\sum_{i=1}^{n} \ln x_{(i)} \\
& +x_{0}^{\alpha} \sum_{i=1}^{n} \frac{\frac{2 \lambda}{x^{\alpha}} \ln \left(\frac{x_{0}}{x_{(i)}}\right)}{1-\lambda+2 \lambda\left(\frac{x_{0}}{x_{(i)}}\right)^{\alpha}}=0 \\
& x_{0}^{\alpha}\left(-\sum_{i=1}^{n}(i-1) \frac{\frac{1}{x^{\alpha}} \ln \left(\frac{x_{0}}{x_{(i)}}\right)}{1-\left(\frac{x_{0}}{x_{(i)}}\right)^{\alpha}}+\sum_{i=1}^{n}(i-1) \frac{\frac{\lambda}{x^{\alpha}} \ln \left(\frac{x_{0}}{x_{(i)}}\right)}{1+\lambda\left(\frac{x_{0}}{x_{(i)}}\right)^{\alpha}}\right. \\
& \left.+\sum_{i=1}^{n}(n-i) \frac{\frac{\lambda}{x^{\alpha}} \ln \left(\frac{x_{0}}{x_{(i)}}\right)}{1-\lambda+\lambda\left(\frac{x_{0}}{x_{(i)}}\right)^{\alpha}}+\sum_{i=1}^{n} \frac{\frac{2 \lambda}{x^{\alpha}} \ln \left(\frac{x_{0}}{x_{(i)}}\right)}{1-\lambda+2 \lambda\left(\frac{x_{0}}{x_{(i)}}\right)^{\alpha}}\right) \\
& +\sum_{i=1}^{n}(n-i) \ln \left(\frac{x_{0}}{x_{(i)}}\right)+\frac{n}{\alpha}+n \ln x_{0}-\sum_{i=1}^{n} \ln x_{(i)}=0
\end{aligned}
$$




$$
\begin{aligned}
& x_{0}^{\alpha}=\frac{\sum_{i=1}^{n} \ln x_{(i)}-\sum_{i=1}^{n}(n-i) \ln \left(\frac{x_{0}}{x_{(i)}}\right)-\frac{n}{\alpha}-n \ln x_{0}}{\left(\begin{array}{c}
\frac{1}{x_{(i)}} \ln \left(\frac{x_{0}}{x_{(i)}}\right) \\
-\sum_{i=1}^{n}(i-1)+\left(\frac{x_{0}}{x_{(i)}}\right)^{\alpha}+\sum_{i=1}^{n}(i-1) \frac{\frac{\lambda}{x_{(i)}} \ln \left(\frac{x_{0}}{x_{(i)}}\right)}{1+\lambda\left(\frac{x_{0}}{x_{(i)}}\right)^{\alpha}} \\
+\sum_{i=1}^{n}(n-i) \frac{\frac{\lambda}{x_{(i)}^{\alpha}} \ln \left(\frac{x_{0}}{x_{(i)}}\right)}{1-\lambda+\lambda\left(\frac{x_{0}}{x_{(i)}}\right)^{\alpha}}+\sum_{i=1}^{n} \frac{\frac{2 \lambda}{x_{(i)}^{\alpha}} \ln \left(\frac{x_{0}}{x_{(i)}}\right)}{1-\lambda+2 \lambda\left(\frac{x_{0}}{x_{(i)}}\right)^{\alpha}}
\end{array}\right)} \\
& \hat{\alpha}_{R S S}=
\end{aligned}
$$

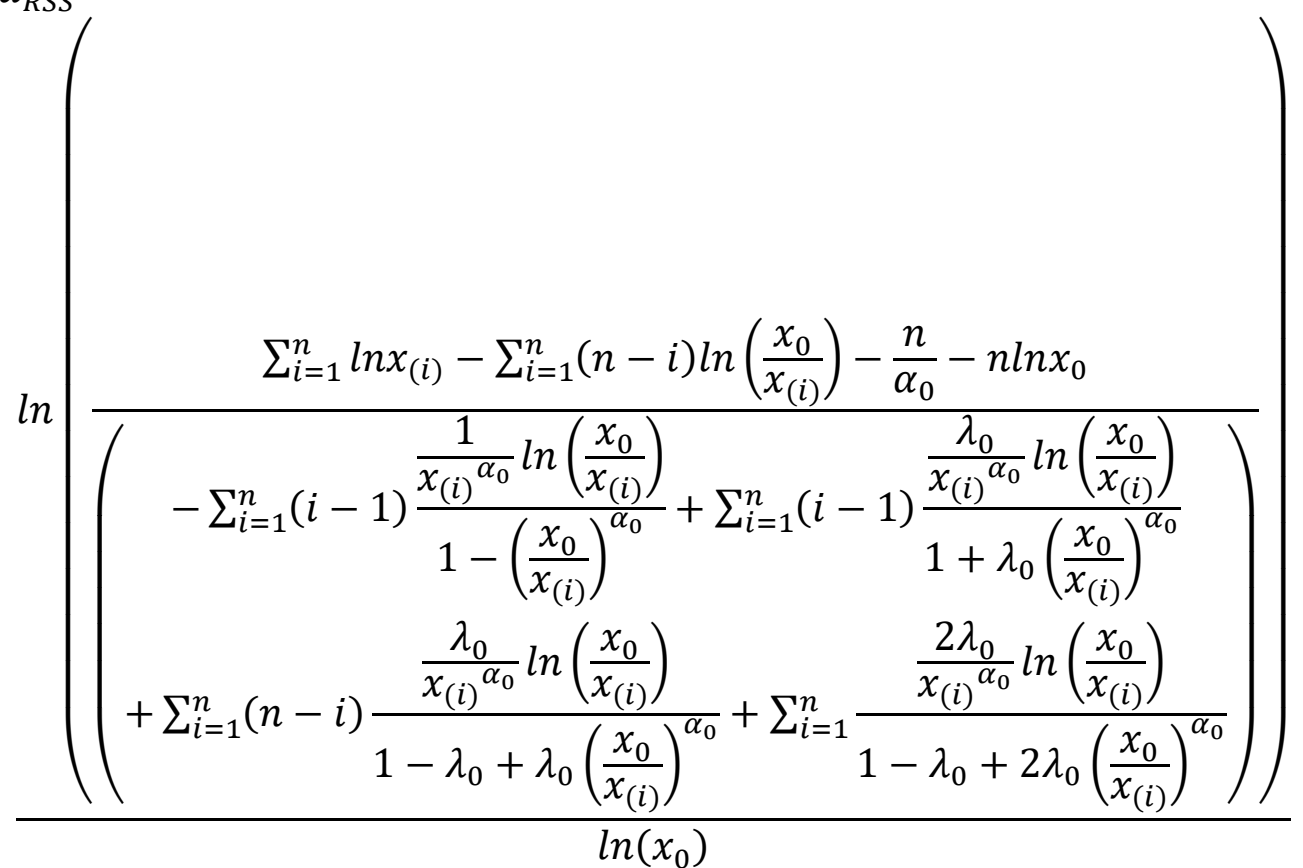

Now we take partial derivative for the equation (37) with respect to $\lambda$ and put it equal to zero

$$
\begin{aligned}
\frac{\partial \ln l f}{\partial \lambda}=\sum_{i=1}^{n}(i-1) \frac{\left(\frac{x_{0}}{x_{(i)}}\right)^{\alpha}}{1+\lambda\left(\frac{x_{0}}{x_{(i)}}\right)^{\alpha}}+\sum_{i=1}^{n}(n-i) \frac{\left(\frac{x_{0}}{x_{(i)}}\right)^{\alpha}-1}{1-\lambda+\lambda\left(\frac{x_{0}}{x_{(i)}}\right)^{\alpha}} & \\
& +\sum_{i=1}^{n} \frac{2\left(\frac{x_{0}}{x_{i}}\right)^{\alpha}-1}{1-\lambda+2 \lambda\left(\frac{x_{0}}{x_{i}}\right)^{\alpha}}=0 \\
-\sum_{i=1}^{n}(i-1) & \frac{\left(\frac{x_{0}}{x_{(i)}}\right)^{\alpha}}{1+\lambda\left(\frac{x_{0}}{x_{(i)}}\right)^{\alpha}} \\
= & \frac{1}{\lambda} \sum_{i=1}^{n}(n-i) \frac{\left(\frac{x_{0}}{x_{(i)}}\right)^{\alpha}-1}{\frac{1}{\lambda}-1+\left(\frac{x_{0}}{x_{(i)}}\right)^{\alpha}}+\frac{1}{\lambda} \sum_{i=1}^{n} \frac{2\left(\frac{x_{0}}{x_{(i)}}\right)^{\alpha}-1}{\lambda}-1+2\left(\frac{x_{0}}{x_{(i)}}\right)^{\alpha}
\end{aligned}
$$




$$
\begin{gathered}
-\sum_{i=1}^{n}(i-1) \frac{\left(\frac{x_{0}}{x_{(i)}}\right)^{\alpha}}{1+\lambda\left(\frac{x_{0}}{x_{(i)}}\right)^{\alpha}} \\
=\frac{1}{\lambda}\left(\sum_{i=1}^{n}(n-i) \frac{\left(\frac{x_{0}}{x_{(i)}}\right)^{\alpha}-1}{\frac{1}{\lambda}-1+\left(\frac{x_{0}}{x_{(i)}}\right)^{\alpha}+\sum_{i=1}^{n} \frac{2\left(\frac{x_{0}}{x_{(i)}}\right)^{\alpha}-1}{\lambda}-1\left(\frac{x_{0}}{x_{(i)}}\right)^{\alpha}}\right) \\
\hat{\lambda}_{R S S}=\frac{\sum_{i=1}^{n}(n-i) \frac{\left(\frac{x_{0}}{x_{(i)}}\right)^{\alpha_{0}}-1}{\frac{1}{\lambda_{0}}-1+\left(\frac{x_{0}}{x_{(i)}}\right)^{\alpha_{0}}+\sum_{i=1}^{n} \frac{1}{\lambda_{0}}-1+2\left(\frac{x_{0}}{x_{(i)}}\right)^{\alpha_{0}}}}{-\sum_{i=1}^{n}(i-1) \frac{\left(\frac{x_{0}}{x_{(i)}}\right)^{\alpha_{0}}}{1+\lambda_{0}\left(\frac{x_{0}}{x_{(i)}}\right)^{\alpha_{0}}}}
\end{gathered}
$$

As for the estimator of the approximate reliability function for this distribution, we obtain it by substituting equations (38) and (39) into equation (3) as follows:

$$
\hat{R}(t)_{R S S}=\left(\frac{x_{0}}{t}\right)^{\widehat{\alpha}_{R S S}}\left(\hat{\lambda}_{R S S}\left(\frac{x_{0}}{t}\right)^{\widehat{\alpha}_{R S S}}-\hat{\lambda}_{R S S}+1\right)
$$

\subsection{Maximum Likelihood Estimation Method (MLE):}

This method of estimation is a common and reliable method, and this method characterized by its accuracy compared to other methods, especially when increasing the sample size.

Let $x_{1}, x_{2}, \ldots, x_{n}$ be a sample of size $n$ for (TP) distribution [8].

$L\left(x_{1}, x_{2}, \ldots, x_{n} ; \alpha, x_{0}, \lambda\right)=\frac{\alpha^{n} x_{0}^{\alpha n}}{\prod_{i=1}^{n} x_{i}^{\alpha+1}} \prod_{i=1}^{n}\left(1-\lambda+2 \lambda\left(\frac{x_{0}}{x_{i}}\right)^{\alpha}\right)$

Taking the natural logarithm for equation (41), we get

$$
\begin{aligned}
\ln L= & n \ln (\alpha)+n \alpha \ln \left(x_{0}\right)-(\alpha+1) \sum_{i=1}^{n} \ln \left(x_{i}\right)+\sum_{i=1}^{n} \ln \left(1-\lambda+2 \lambda\left(\frac{x_{0}}{x_{i}}\right)^{\alpha}\right) \\
= & n \ln (\alpha)+n \alpha \ln \left(x_{0}\right)-\alpha \sum_{i=1}^{n} \ln \left(x_{i}\right)-\sum_{i=1}^{n} \ln \left(x_{i}\right) \\
& +\sum_{i=1}^{n} \ln \left(1-\lambda+2 \lambda\left(\frac{x_{0}}{x_{i}}\right)^{\alpha}\right)
\end{aligned}
$$

By taking partial derivative for equation (42) with respect to $\alpha$ and equal to zero

$$
\begin{aligned}
\frac{\partial \ln L}{\partial \alpha} & =\frac{n}{\alpha}+n \ln \left(x_{0}\right)-\sum_{i=1}^{n} \ln \left(x_{i}\right)+\sum_{i=1}^{n} \frac{2 \lambda\left(\frac{x_{0}}{x_{i}}\right)^{\alpha} \ln \left(\frac{x_{0}}{x_{i}}\right)}{1-\lambda+2 \lambda\left(\frac{x_{0}}{x_{i}}\right)^{\alpha}}=0 \\
\frac{n}{\alpha} & =\sum_{i=1}^{n} \ln \left(x_{i}\right)-n \ln \left(x_{0}\right)-\sum_{i=1}^{n} \frac{2 \lambda\left(\frac{x_{0}}{x_{i}}\right)^{\alpha} \ln \left(\frac{x_{0}}{x_{i}}\right)^{\alpha}}{1-\lambda+2 \lambda\left(\frac{x_{0}}{x_{i}}\right)^{\alpha}}
\end{aligned}
$$




$$
\hat{\alpha}_{M L E}=\frac{n}{\sum_{i=1}^{n} \ln \left(x_{i}\right)-n \ln \left(x_{0}\right)-\sum_{i=1}^{n} \frac{2 \lambda_{0}\left(\frac{x_{0}}{x_{i}}\right)^{\alpha_{0}} \ln \left(\frac{x_{0}}{x_{i}}\right)}{1-\lambda_{0}+2 \lambda_{0}\left(\frac{x_{0}}{x_{i}}\right)^{\alpha_{0}}}}
$$

By taking partial derivative for equation (42) with respect to $\lambda$ and equal to zero

$$
\begin{aligned}
& \frac{\partial \ln \mathrm{L}}{\partial \lambda}=\sum_{i=1}^{n} \frac{2\left(\frac{x_{0}}{x_{i}}\right)^{\alpha}-1}{1-\lambda+2 \lambda\left(\frac{x_{0}}{x_{i}}\right)^{\alpha}} \\
& \sum_{i=1}^{n} \frac{2\left(\frac{x_{0}}{x_{i}}\right)^{\alpha}}{1-\lambda+2 \lambda\left(\frac{x_{0}}{x_{i}}\right)^{\alpha}}-\sum_{i=1}^{n} \frac{1}{1-\lambda+2 \lambda\left(\frac{x_{0}}{x_{i}}\right)^{\alpha}}=0 \\
& \frac{1}{\lambda} \sum_{i=1}^{n} \frac{1}{\frac{1}{\lambda}-1+2\left(\frac{x_{0}}{x_{i}}\right)^{\alpha}}=\sum_{i=1}^{n} \frac{2\left(\frac{x_{0}}{x_{i}}\right)^{\alpha}}{1-\lambda+2 \lambda\left(\frac{x_{0}}{x_{i}}\right)^{\alpha}} \\
& \hat{\lambda}_{M L E}=\frac{\sum_{i=1}^{n} \frac{1}{\frac{1}{\lambda_{0}}-1+2\left(\frac{x_{0}}{x_{i}}\right)^{\alpha_{0}}}}{\sum_{i=1}^{n} \frac{2\left(\frac{x_{0}}{x_{i}}\right)^{\alpha_{0}}}{1-\lambda_{0}+2 \lambda_{0}\left(\frac{x_{0}}{x_{i}}\right)^{\alpha_{0}}}}
\end{aligned}
$$

As for the estimator of the reliability function for this distribution, we obtain it by substituting equations (43) and (44) into equation (3) as follows:

$\hat{R}(t)_{M L E}=\left(\frac{x_{0}}{t}\right)^{\hat{\alpha}_{M L E}}\left(\hat{\lambda}_{M L E}\left(\frac{x_{0}}{t}\right)^{\hat{\alpha}_{M L E}}-\hat{\lambda}_{M L E}+1\right)$

\section{Experiments and Results:}

In this section, we review the simulation steps in terms of selecting sample sizes, real values of parameters and life time values that were used to estimate reliability:

1. The sample size is $n=10,30,70,100$, and the sample which is replicated $N=1000$.

2. Several values of the shape and the transmuted parameters $(\alpha, \lambda)$ are shown in Table (1) below:

Table 1-The default value for parameters

\begin{tabular}{|c|c|c|c|c|}
\hline \multicolumn{2}{|c|}{ Cases } & $C_{1}$ & $C_{2}$ & $C_{3}$ \\
\hline \multirow{2}{*}{ parameters } & $\alpha$ & 4 & 1.5 & 1 \\
\cline { 2 - 5 } & $\lambda$ & -1 & 0.1 & 0.5 \\
\hline
\end{tabular}

3. In all cases $C_{1}, C_{2}$, and $C_{3}$ the life time for estimating reliability is chosen $x_{0} \leq t \leq x_{(n)}$, such that, $t_{(i)}=0.1,0.2, \ldots \leq x_{(n)}$.

4. At this stage, random data is generated of (TP) distribution by equation (4) and using MATLAB language version R2012b.

5. At this stage we find the value of parameter and reliability estimated according to the equations(10),(11),(12),(16),(18),(19),(24),(25),(26),(30),(32),(33),(38),(39),(40),(43),(44)and (45) .

6. Finally, comparison between the estimators is done by $\operatorname{MSE}(\hat{\beta})=\frac{\sum_{i=1}^{N}\left(\widehat{\beta}_{i}-\beta\right)^{2}}{N}$, where $(\hat{\beta})$ is an estimator for parameter $(\beta)$. 
The next Tables 2,3 and 4 show the empirical value for estimation of parameters and reliability function.

Table 2-Estimated values for $(R)$ and $(\alpha, \lambda)$ using $\left(C_{1}: R=0.56054\right)$

\begin{tabular}{|c|c|c|c|c|c|c|c|}
\hline \multirow{2}{*}{ Methods } & \multirow{2}{*}{$\mathrm{n}$} & \multicolumn{3}{|c|}{ Mean Estimated Values } & \multicolumn{3}{|c|}{ MSE } \\
\hline & & $\hat{\alpha}$ & $\hat{\lambda}$ & $\hat{R}$ & $\hat{\alpha}$ & $\hat{\lambda}$ & $\widehat{R}$ \\
\hline MOM & \multirow{6}{*}{10} & 3.72277 & -0.24730 & 0.54652 & 0.07685 & 0.56654 & 0.00039 \\
\hline MM & & 2.86833 & -1.81925 & 0.67598 & 1.28065 & 0.67118 & 0.02665 \\
\hline LM & & 2.48736 & -2.11013 & 0.48752 & 2.28807 & 1.23239 & 0.13427 \\
\hline PER & & 3.37621 & -1.43156 & 0.61044 & 0.38910 & 0.18624 & 0.00498 \\
\hline RSS & & 2.85868 & -1.66261 & 0.66773 & 1.30260 & 0.43905 & 0.02298 \\
\hline MLE & & 5.23822 & -1.66195 & 0.53467 & 1.53319 & 0.43817 & 0.00133 \\
\hline MOM & \multirow{6}{*}{30} & 3.82981 & -1.27414 & 0.29960 & 0.02896 & 0.07515 & 0.00028 \\
\hline MM & & 3.20095 & -1.50389 & 0.62725 & 0.63847 & 0.25390 & 0.00889 \\
\hline LM & & 2.89237 & -1.60037 & 0.32119 & 1.22684 & 0.36045 & 0.04004 \\
\hline PER & & 3.78210 & -1.13547 & 0.57461 & 0.04747 & 0.01835 & 0.00039 \\
\hline RSS & & 3.98640 & -1.22689 & 0.56780 & 0.00018 & 0.05148 & 0.00010 \\
\hline MLE & & 3.60794 & -1.13183 & 0.58361 & 0.15370 & 0.01737 & 0.00106 \\
\hline MOM & \multirow{6}{*}{70} & 3.85182 & -1.20419 & 0.23873 & 0.02195 & 0.04169 & 0.00014 \\
\hline MM & & 4.06634 & -1.03555 & 0.55890 & 0.00440 & 0.00126 & $0.53783 * 10^{-5}$ \\
\hline LM & & 3.86621 & -1.04340 & 0.13521 & 0.01789 & 0.00188 & 0.00019 \\
\hline PER & & 3.82503 & -0.98321 & 0.29358 & 0.03061 & 0.00028 & 0.00005 \\
\hline RSS & & 4.00200 & -1.14075 & 0.23341 & $0.40290 * 10^{-5}$ & 0.01981 & 0.00001 \\
\hline MLE & & 4.35358 & -0.9109 & 0.36907 & 0.12502 & 0.00793 & 0.00032 \\
\hline MOM & \multirow{6}{*}{100} & 4.03570 & -0.83677 & 0.22803 & 0.00127 & 0.02664 & 0.00003 \\
\hline MM & & 4.00239 & -1.03502 & 0.23179 & $0.57524 * 10^{-5}$ & 0.00122 & $0.71667 * 10^{-6}$ \\
\hline LM & & 4.12611 & -0.96316 & 0.06512 & 0.01590 & 0.00135 & 0.00007 \\
\hline PER & & 4.12690 & -0.98711 & 0.37724 & 0.01610 & 0.00016 & 0.00004 \\
\hline RSS & & 3.99925 & -0.92980 & 0.38024 & $0.55311 * 10^{-6}$ & 0.00492 & $0.57134 * 10^{-5}$ \\
\hline MLE & & 3.69776 & -0.94278 & 0.29719 & 0.09134 & 0.00327 & 0.00015 \\
\hline
\end{tabular}

Table 3-Estimated values for $(R)$ and $(\alpha, \lambda)$ using $\left(C_{2}: R=0.12193\right)$

\begin{tabular}{|c|c|c|c|c|c|c|c|}
\hline \multirow[t]{2}{*}{ Methods } & \multirow[t]{2}{*}{$\mathrm{n}$} & \multicolumn{3}{|c|}{ Mean Estimated Values } & \multicolumn{3}{|c|}{ MSE } \\
\hline & & $\hat{\alpha}$ & $\hat{\lambda}$ & $\hat{R}$ & $\hat{\alpha}$ & $\hat{\lambda}$ & $\hat{R}$ \\
\hline LM & \multirow{4}{*}{10} & 0.73975 & 0.19392 & 0.30527 & 0.57797 & 0.00882 & 0.03445 \\
\hline PER & & 0.83693 & 0.17575 & 0.36077 & 0.43965 & 0.00573 & 0.01968 \\
\hline RSS & & 0.90193 & 0.15328 & 0.34411 & 0.35768 & 0.00283 & 0.01503 \\
\hline MLE & & 0.89462 & 0.18277 & 0.34149 & 0.36647 & 0.00685 & 0.01432 \\
\hline LM & \multirow{4}{*}{30} & 0.93430 & 0.14772 & 0.22452 & 0.32001 & 0.00227 & 0.01363 \\
\hline PER & & 1.15235 & 0.12299 & 0.26205 & 0.12085 & 0.00052 & 0.00336 \\
\hline RSS & & 1.11096 & 0.10373 & 0.27312 & 0.15135 & 0.00001 & 0.00491 \\
\hline MLE & & 1.17519 & 0.13385 & 0.25623 & 0.10549 & 0.00114 & 0.00265 \\
\hline $\mathrm{LM}$ & \multirow{4}{*}{70} & 1.42540 & 0.10343 & 0.14740 & 0.00556 & 0.00001 & 0.00015 \\
\hline PER & & 1.71230 & 0.08796 & 0.23527 & 0.04507 & 0.00014 & 0.00074 \\
\hline RSS & & 1.70284 & 0.10099 & 0.14499 & 0.04114 & $0.98305 * 10^{-6}$ & 0.00049 \\
\hline MLE & & 1.68063 & 0.08734 & 0.05126 & 0.03263 & 0.00016 & 0.00012 \\
\hline LM & \multirow{4}{*}{100} & 1.57141 & 0.09711 & 0.05897 & 0.00509 & $0.83307 * 10^{-5}$ & 0.00005 \\
\hline PER & & 1.53628 & 0.10255 & 0.05695 & 0.00131 & $0.65034 * 10^{-5}$ & $0.75572 * 10^{-5}$ \\
\hline RSS & & 1.39296 & 0.09913 & 0.05558 & 0.01145 & $0.74377 * 10^{-6}$ & 0.00006 \\
\hline MLE & & 1.58805 & 0.09919 & 0.11295 & 0.00775 & $0.65335 * 10^{-6}$ & 0.00007 \\
\hline
\end{tabular}


Table 4- Estimated values for $(R)$ and $(\alpha, \lambda)$ using $\left(C_{3}: R=0.10352\right)$

\begin{tabular}{|c|c|c|c|c|c|c|c|}
\hline \multirow{2}{*}{ Methods } & \multirow{2}{*}{$\mathrm{n}$} & \multicolumn{3}{|c|}{ Mean Estimated Values } & \multicolumn{3}{|c|}{ MSE } \\
\hline & & $\hat{\alpha}$ & $\hat{\lambda}$ & $\hat{R}$ & $\hat{\alpha}$ & $\hat{\lambda}$ & $\hat{R}$ \\
\hline LM & \multirow{4}{*}{10} & 0.47524 & 0.8560 & 0.21287 & 0.27536 & 0.12292 & 0.01304 \\
\hline PER & & 0.42657 & 0.93068 & 0.27774 & 0.32882 & 0.18548 & 0.01530 \\
\hline RSS & & 0.34417 & 0.87600 & 0.35307 & 0.43011 & 0.14138 & 0.04020 \\
\hline MLE & & 0.46512 & 0.80456 & 0.28086 & 0.28609 & 0.09276 & 0.01590 \\
\hline LM & \multirow{4}{*}{30} & 0.61355 & 0.67701 & 0.16839 & 0.14933 & 0.03133 & 0.00604 \\
\hline PER & & 0.68005 & 0.60771 & 0.20627 & 0.10236 & 0.01160 & 0.00403 \\
\hline RSS & & 0.75106 & 0.55404 & 0.19361 & 0.06196 & 0.00292 & 0.00249 \\
\hline MLE & & 0.68149 & 0.62521 & 0.20287 & 0.10144 & 0.01567 & 0.00359 \\
\hline $\mathrm{LM}$ & \multirow{4}{*}{70} & 0.94907 & 0.51307 & 0.12318 & 0.00259 & 0.00017 & 0.00008 \\
\hline PER & & 1.15729 & 0.42376 & 0.16639 & 0.02474 & 0.00581 & 0.00032 \\
\hline RSS & & 1.13740 & 0.48354 & 0.09385 & 0.01887 & 0.00027 & 0.00029 \\
\hline MLE & & 1.06863 & 0.47535 & 0.17407 & 0.00471 & 0.00060 & 0.00009 \\
\hline $\mathrm{LM}$ & \multirow{4}{*}{100} & 1.04866 & 0.48895 & 0.04402 & 0.00236 & $0.12191 * 10^{-3}$ & 0.00002 \\
\hline PER & & 1.03902 & 0.51961 & 0.02482 & 0.00152 & 0.00038 & 0.00001 \\
\hline RSS & & 0.93972 & 0.48880 & 0.02527 & 0.00363 & $0.12521 * 10^{-3}$ & 0.00002 \\
\hline MLE & & 1.05076 & 0.47643 & 0.14971 & 0.00257 & 0.00055 & 0.00003 \\
\hline
\end{tabular}

\section{Conclusion:}

From the results in Tables 2, 3 and 4, we note the following:

- In the second and third cases $\left(C_{2}, C_{3}\right)$, we note that the parameters $(\alpha, \lambda)$ and the reliability function cannot be estimated for the two methods, namely MOM, and MM, because there is a condition in the estimate formulas, which is $\alpha>2$.

- In the first case $\left(C_{1}\right)$, we note that RSS is the best in estimating $(\alpha)$ in all cases, while for $n=10 \mathrm{MOM}$ is the best in estimating the $(\alpha)$,

and PER is the best in estimating $(\lambda)$ in all cases, while for $n=30$ MLE is the best in estimating the $(\lambda)$.

For the reliability function of the distribution the MM is best when $n=70,100$, while if $n=10$, then MOM is the best as well as if $n=30$, then RSS is the best.

- In the second case $\left(C_{2}\right)$, in the estimation of $(\alpha)$, the RSS is best when $n=10$, and if $n=30$, then MLE is the best, while if $n=70$, then the LM is the best as well as when $n=100$ the PER is the best.

The RSS is the best in estimating $(\lambda)$ in all cases, while for $n=100$ the MLE is the best in estimating the $(\lambda)$.

In estimating reliability the MLE is best in all cases, while for $n=100$ the PER is the best.

- In the third case $\left(C_{3}\right)$, we note that the LM is the best in estimating $(\alpha)$ when $n=10,70$, however in $n=30$ the RSS is the best, and when $n=100$ the PER is the best. The LM is the best in estimating $(\lambda)$ when $n=70,100$, but when $n=10$ the MLE is the best, and when $n=30$ the RSS is the best.

In estimating reliability the LM is best when $n=10,70$, but when $n=30$ the RSS is the best and when $n=100$ the PER is the best. 


\section{References:}

[1] C. Press, "The New Theories of Economics Author ( s ): Vilfredo Pareto Source: Journal of Political Economy, vol . 5, no . 4 ( Sep ., 1897 ), pp . 485-502 Published by: The University of Chicago Press Stable URL: http://www.jstor.org/stable/1821012 .," vol. 5, no. 4, pp. 485-502, 2013.

[2] W. T. Shaw and I. R. Buckley, "The alchemy of probability distributions: Beyond gram-charlier \& cornish-fisher expansions, and skew-normal or kurtotic-normal distributions," Submitt. Feb, vol. 7, pp. 64, 2007.

[3] G. R. Aryal and C. P. Tsokos, "Transmuted Weibull Distribution: A Generalization of the Weibull Probability Distribution," Eur. J. Pure Appl. Math., vol. 4, no. 2, pp. 89-102, 2011.

[4] G. R. Aryal, "Transmuted Log-Logistic Distribution," J. Stat. Appl. Probab., vol. 2, no. 1, pp. 11-20, 2013. doi: 10.12785/jsap/020102.

[5] M. A. ul Haq, "Transmuted Exponentiated Inverse Rayleigh Distribution," J. Stat. Appl. Probab., vol. 5, no. 2, pp. 337-343, 2016.doi: 10.18576/jsap/050215.

[6] R. V. VARDHAN and S. BALASWAMY, "Transmuted New Modified Weibull Distribution," Math. Sci. Appl. E-Notes, vol. 4, no. 1, pp. 125-135, 2016. doi: 10.36753/mathenot.421421.

[7] M. Elgarhy, I. Elbatal, L. S. Diab, H. K. Hwas, and A. W. Shawki, "Transmuted Generalized Quasi Lindley Distribution," Int. J. Sci. Eng. Sci. ISSN (Online, vol. 1, no. 7, pp. 1-8, 2017.http://ijses.com/wp-content/uploads/2017/07/105-IJSES-V1N6.pdf.

[8] F. Merovci and L. Puka, "Transmuted Pareto distribution," ProbStat Forum, vol. 07. pp. 1-11, 2014.

[9] M. A. Mohammed Salih and J. H. Eidi, "Using Simulation to Estimate Reliability for Transmuted Inverse Exponential Distribution," 1st Int. Sci. Conf. Comput. Appl. Sci. CAS .pp. 16-21, 2019. doi: 10.1109/CAS47993.2019.9075709.

[10] A. C. Cohen and B. J. Whitten, "Modified Moment and Maximum Likelihood Estimators for Parameters of the Three-Parameter-Gamma Distribution," Commun. Stat. - Simul. Comput., vol. 11, no. 2, pp. 197-216, 1982. doi: 10.1080/03610918208812254.

[11] M. A. M. Salih and A. J. Kamees, "Reliability Estimation for two parameters log-logistic Distribution,"no. January 2017, 2019.

[12] J. R. M. Hosking, "of Distributions using Linear Analysis and Estimation of Order Statistics Combinations by equating the Yet moment-based The alternative approach described here is based on quantities," J. R. Stat. Soc., vol. 52, no. 1, pp. 105-124, 1990.

[13] D. Aydoğan, M. Kankal, and H. Önsoy, "Regional flood frequency analysis for Çoruh Basin of Turkey with L-moments approach," J. Flood Risk Manag., vol. 9, no. 1, pp. 69-86, 2016. doi: 10.1111/jfr3.12116.

[14] S. H. Bhatti, S. Hussain, T. Ahmad, M. Aslam, M. Aftab, and M. A. Raza, "Efficient estimation of Pareto model: Some modified percentile estimators," PLoS One, vol. 13, no. 5, pp. 1-15, 2018. doi: 10.1371/journal.pone.0196456.

[15] M. H. Samuh, A. I. Al-omari, and N. Koyuncu, "Weibull-Pareto Distribution using Ranked set," 2020. 